AHA!

Miszellen zur Gartengeschichte und Gartendenkmalpflege

\title{
Stürmische Zeiten in Thammenhain
}

\author{
Nora Kindermann
}

G leich drei große Stürme, zuletzt die beiden Sturmtiefs Xavier und Herwart im Oktober, hatten im Jahr 2017 den Thammenhainer Schlosspark zum Teil mit Orkanböen heimgesucht. Etliche Altbäume konnten diesem starken Winddruck nicht standhalten. So kam es zu zahlreichen Abbrüchen von Starkästen und ganzen Kronenzwieseln sowie leider auch zum kompletten Verlust von Altbäumen durch Windwurf. Dies ist besonders im Bereich der Grotte eklatant, da diese nun ihres Kronenschirmes beraubt ist. Das nunmehr vierte Parkseminar vom 23. bis zum 26. November 2017 stand daher ganz im Zeichen der Beseitigung von Sturmschäden.

Damit waren zunächst Aufräumarbeiten, vor allem im Bereich des Pleasuregrounds und der Grotte, verbunden. Geschädigte Gehölze mit großen Stammwunden durch Starkastausrisse mussten gefällt, sowie das Rau- und Stammholz beräumt werden. Darüberhinaus wurde im Bereich der Grotte mit der Beräumung eines großen Ahornstubbens begonnen. Anknüpfend an die im Jahr 2016 erfolgten Pflanzarbeiten wurden zwei junge Traubeneichen als Ersatz für die verloren gegangenen Altbäume eingebracht. Diese stehen in neu aufgepflanzten Strauchgruppen, die einen ersten Ansatz zur Wiederetablierung einer Strauchschicht im Pleasureground bilden. Wolliger Schneeball (Viburnum lantana), Winter-Heckenkirsche (Lonicera purpusii) und Schneebeere (Symphoricarpos albus) werden den Park in vielerlei Hinsicht bereichern und nicht zuletzt durch ihre Herbstfärbung Akzente setzen.

Des Weiteren wurden die Arbeiten im an die Obstbaumwiese angrenzenden Gehölz- bereich weitergeführt und dem dortigen Spitz-Ahornaufwuchs erneut erfolgreich der Kampf angesagt. Die in den vergangenen Jahren freigestellten Bereiche befreite eine der Arbeitsgruppen mittels eines Kontrollgangs erneut von einjährigen Spitz-Ahornsämlingen. In den angrenzenden Bereichen beseitigten sie den Stangenaufwuchs, so dass der hainartige Altbaumbestand wieder erlebbar wird und die vereinzelt darin vorhandenen Sträucher nun eine Möglichkeit der Entwicklung und Ausbreitung haben.

Eine neue Attraktion erhielt der Park durch die Wiederinpflegenahme der Insel im Schlossteich. Diese ist nun nicht mehr nur eine grüne Gehölzmasse inmitten des Wassers, sondern wieder als gestalteter Glanzpunkt der Anlage mit einzelnen Baumgestalten wie der mächtigen Solitäreiche in der Mitte erkennbar. Mittels des Einsatzes von Booten und Wathosen war es möglich, den Aufwuchs zu entfernen und von der Insel wegzubringen. Damit bieten sich nun rings um den Schlossteich immer wieder neue Blickperspektiven auf die Solitäreiche und zum gegenüberliegenden Ufer durch den transparenter gewordenen $\mathrm{Ge}$ hölzsaum der Insel.

Erneut war ein Baumklettererteam im Einsatz, das - bedingt durch die Stürme viel loses Totholz aus den Kronen holen musste und so einen wesentlichen Beitrag zur Gewährleistung der Verkehrssicherheit während des Seminars leistete.

Die fachliche Anleitung übernahm in gewohnter Weise Henrike Schwarz vom Landesamt für Denkmalpflege Sachsen, Sachgebiet Gartendenkmalpflege. Neben den Studierenden unseres Instituts nahm in 


\section{Stürmische Zeiten in Thammenhain}

diesem Jahr Dr. Justyna Jaworek vom Institut für Landschaftsarchitektur an der Naturwissenschaftlichen Universität Breslau am Parkseminar teil. Im Rahmen der Internationalisierung der Lehrinhalte der Lehrveranstaltung Gartendenkmalpflege soll die Zusammenarbeit zwischen dem Institut für Landschaftsarchitektur an der TU Dresden und dem Institut für Landschaftsarchitektur an der Naturwissenschaftlichen Universität Breslau weiter ausgebaut werden. Es ist geplant, ein vergleichbares Parkseminar im Hirschberger Tal durchzuführen, bei dem deutsche und polnische Studierende gemeinsam einen Beitrag zur Pflege und Erhaltung historischer Parkanlagen leisten.
Ihre Teilnahme an diesem Einsatz war ein erster Schritt zur Realisierung dieses Vorhabens.

Wir danken an dieser Stelle der HerbertHeise-Stiftung für Gartenkunst und Landschaftskultur für die erneute Förderung, die uns auch in diesem Jahr einen reibungslosen Ablauf der Arbeiten ermöglichte. Zudem sei allen Teilnehmern von 1 bis 77 Jahren gedankt, die im Park tätig waren und die auch der Dauerregen am zweiten Arbeitstag nicht schrecken konnte. Nicht zuletzt danken wir unseren Gastgebern, Rüdiger und Elisabeth von Schönberg, die uns großzügig aufnahmen und deren Gastfreundschaft wir für vier Tage genießen durften.

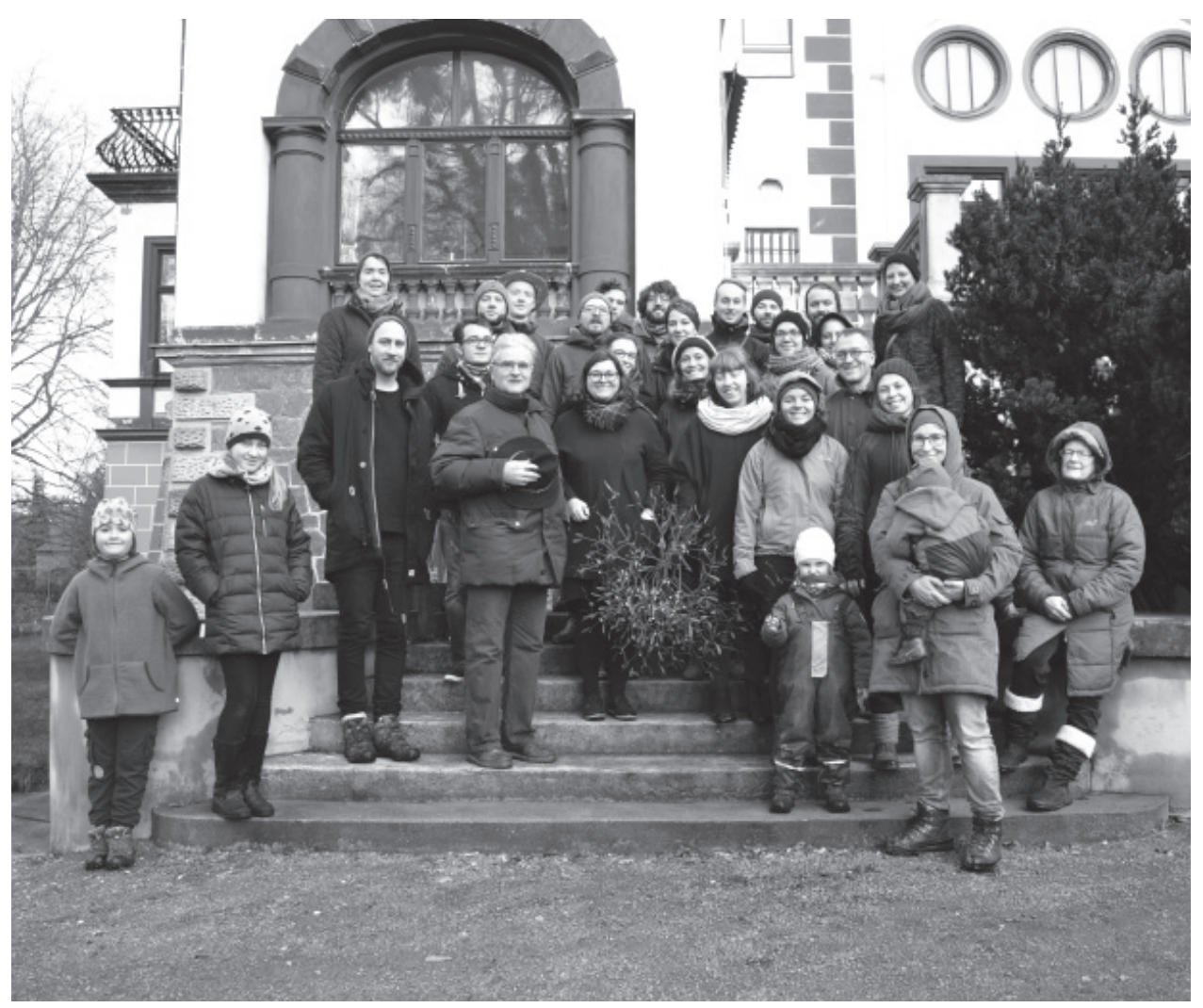

1 | Die Teilnehmer des Parkseminars nach erfolgreicher Arbeit, Fotografie, Ivo Kindermann 2017. 


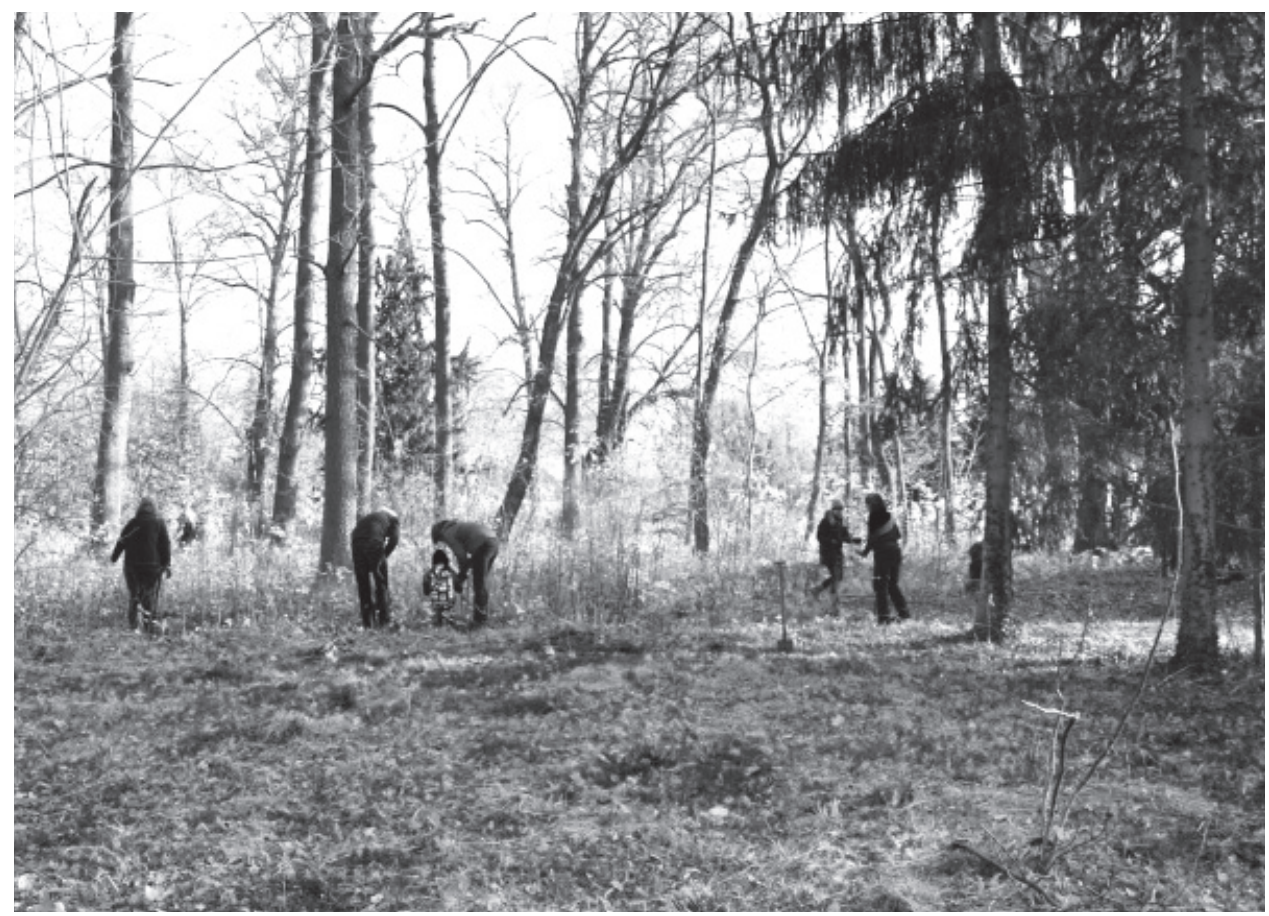

2 | Beseitigung des Sämlingaufwuchses zwischen Gästehäusern und Obstwiese, Fotografie, Ivo Kindermann 2017.

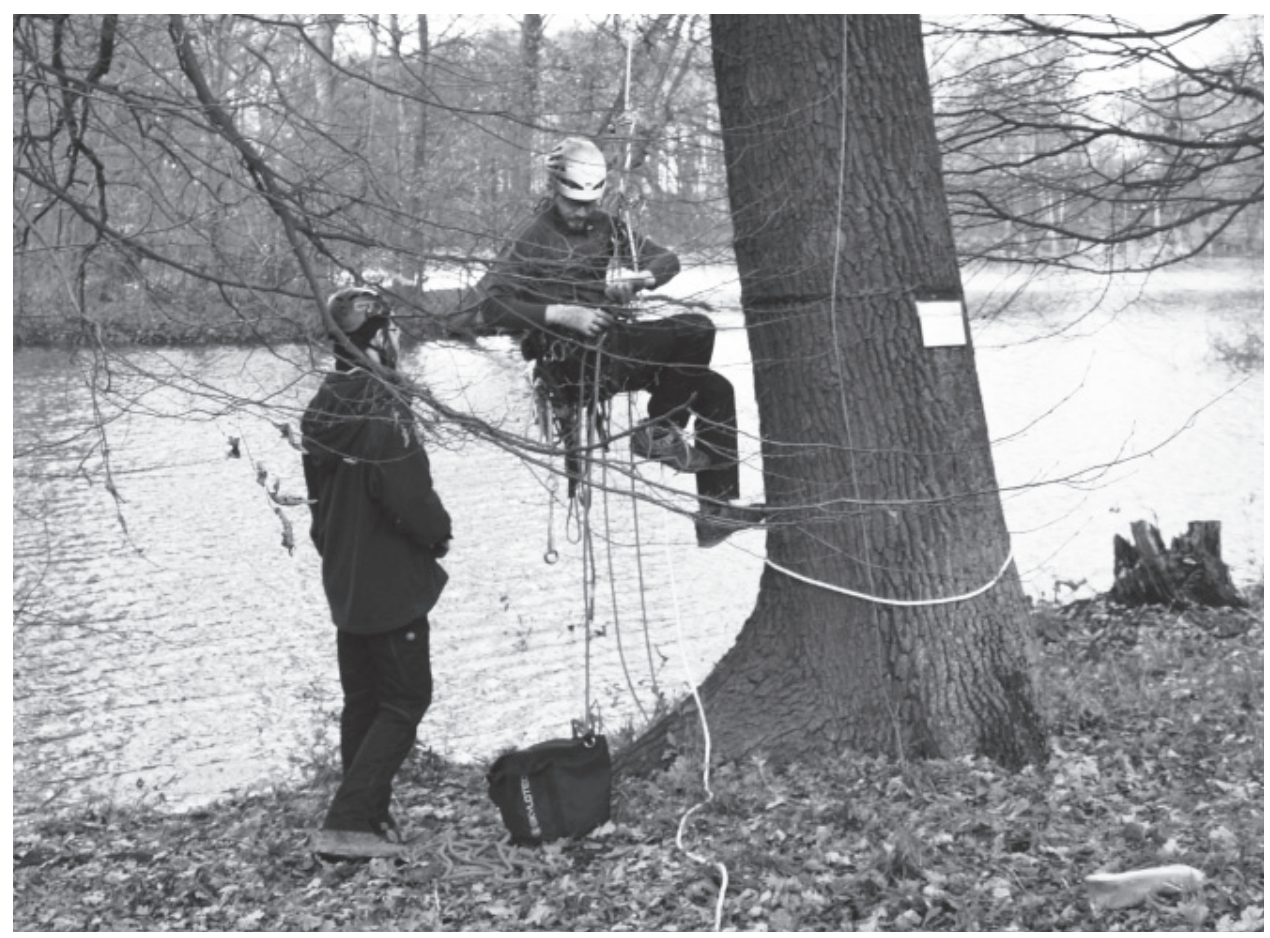

3 | Die Baumkletterer sorgten für die Sicherheit der Seminaristen, Fotografie, Ivo Kindermann 2017. 


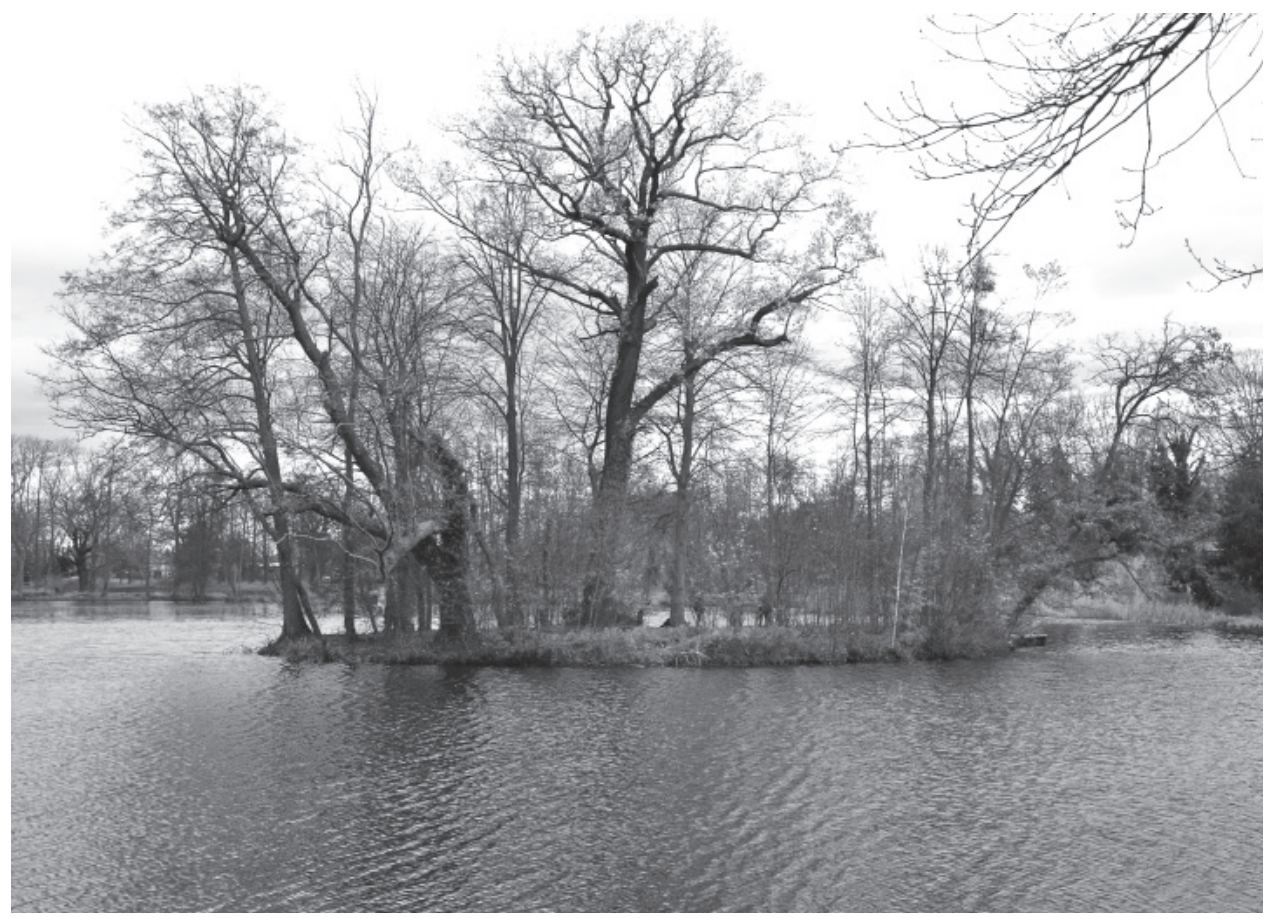

4 | Die zugewachsene Insel im Schlossteich zu Beginn des Seminars, Fotografie, Jenny Pfriem 2017.

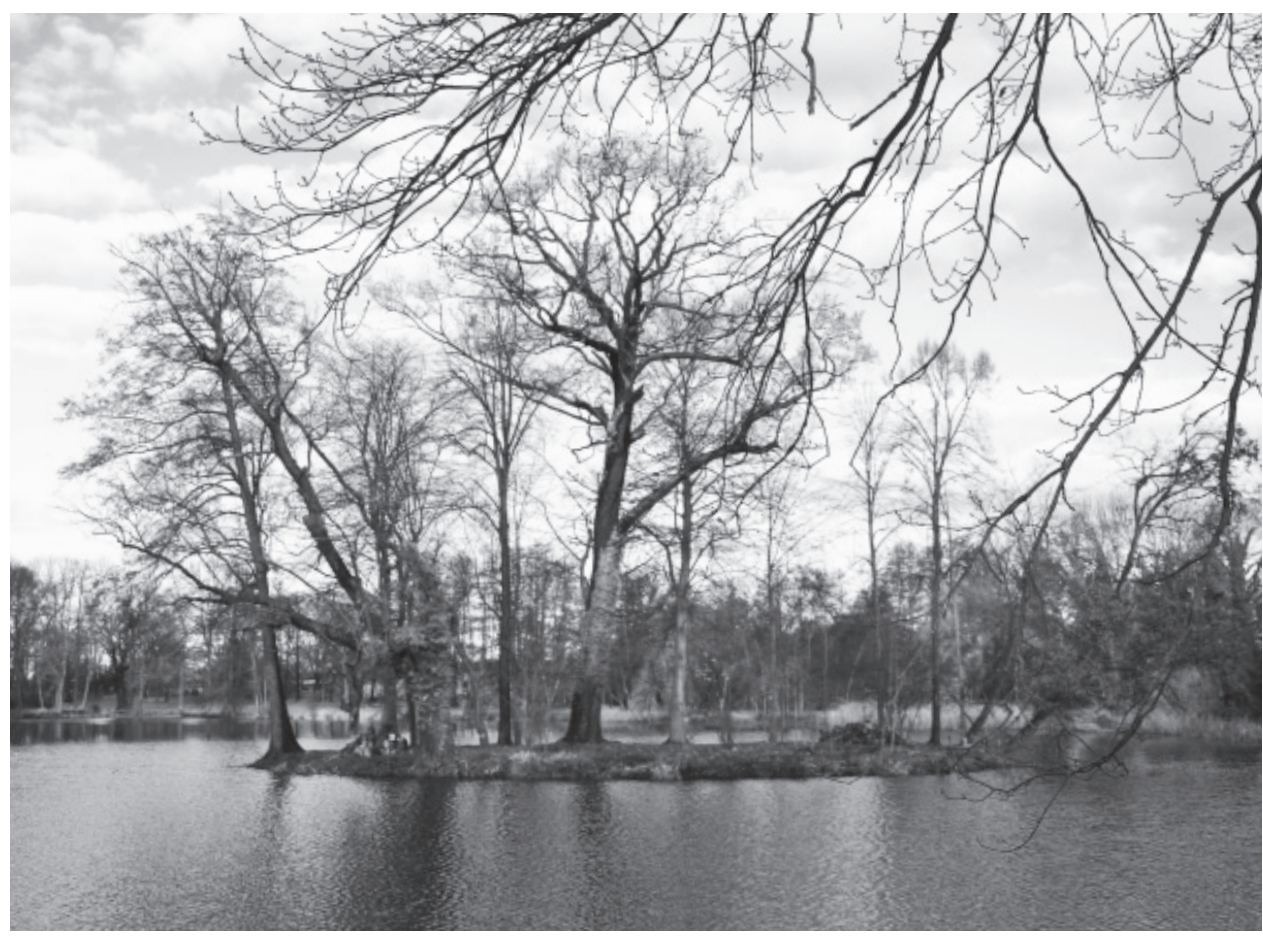

5 | Die freigestellte Insel im Schlossteich nach Abschluss der Maßnahmen, Fotografie, Jenny Pfriem 2017. 